\section{Can molecular imaging techniques identify biomarkers for neuropsychiatric disorders?}

\author{
Podem as técnicas de \\ imagem molecular \\ identificar biomarcadores \\ nos distúrbios \\ neuropsiquiátricos?
}

The phenomenological paradigm used for neuropsychiatric diagnosis is still hegemonic in the currently adopted classifications. This paradigm, however, has the particular caveat of not including any information from biological or pathophysiological mechanisms. With recent advances in the methods for clinical neurosciences investigations, promising new approaches to investigate the pathophysiology of neuropsychiatric disorders (NPD) have become available and are now leading to studies that attempt to unravel the neurobiological underpinnings of these diseases. Advances in molecular imaging techniques, such as positron emission tomography (PET) and single photon emission computerized tomography (SPECT), have made important contributions to the understanding of the pathophysiology of NPD. These techniques use radiolabeled tracers, which allow direct investigation of neuroreceptors in vivo at any stage of the NPD. More sensitive than neuroanatomical imaging techniques, molecular imaging approaches are able to identify subtle cerebral pathophysiological changes before neurostructural abnormalities take place.

One of the major goals of molecular imaging research has been the identification of biomarkers, which are defined as the characteristics that are objectively measured and can differentiate normal biologic processes from pathogenic processes. These approaches are at a privileged condition to potentially provide accurate and early NPD recognition, evaluate disease progression, and monitor treatment efficacy. In recent years, attention has been turned to individuals at risk of developing NPD aiming to prevent the progression of these disorders. Biomarkers, as indicators of underlying biological processes, offer the potential to identify asymptomatic individuals who will develop the NPD in the following years.

An example of the use of PET and SPECT to identify biomarkers of NPD are the dopamine transporter (DAT) radiotracers that evaluate dopamine neuronal loss in Parkinson's disease (PD). The DAT are located in the axon terminals of nigrostriatal dopamine neurons located in the striatum and are expected to decrease with the dopaminergic neurodegeneration observed in PD. Several receptor-imaging studies have shown that $P D$ patients have decreased DAT when compared to matched controls (for review see Ravina et al., 2005). ${ }^{1}$ We have recently performed a study with similar results in Brazil. ${ }^{2-3}$ The studies have consistently shown a negative correlation between DAT density and PD progression. Figure 1 shows several SPECT brain images of PD patients at the level of the striatum (higher color intensity means higher DAT density). The images were acquired from patients with increasing PD severity as measured with Hoehn \& Yahr scale. The decrease in DAT density with the progression of PD is evident in these scans.

Current diagnosis of PD still relies on subjective clinical criteria, which include bradykinesia, and at least one of other three core symptoms (resting tremor, postural instability, and rigidity). This 


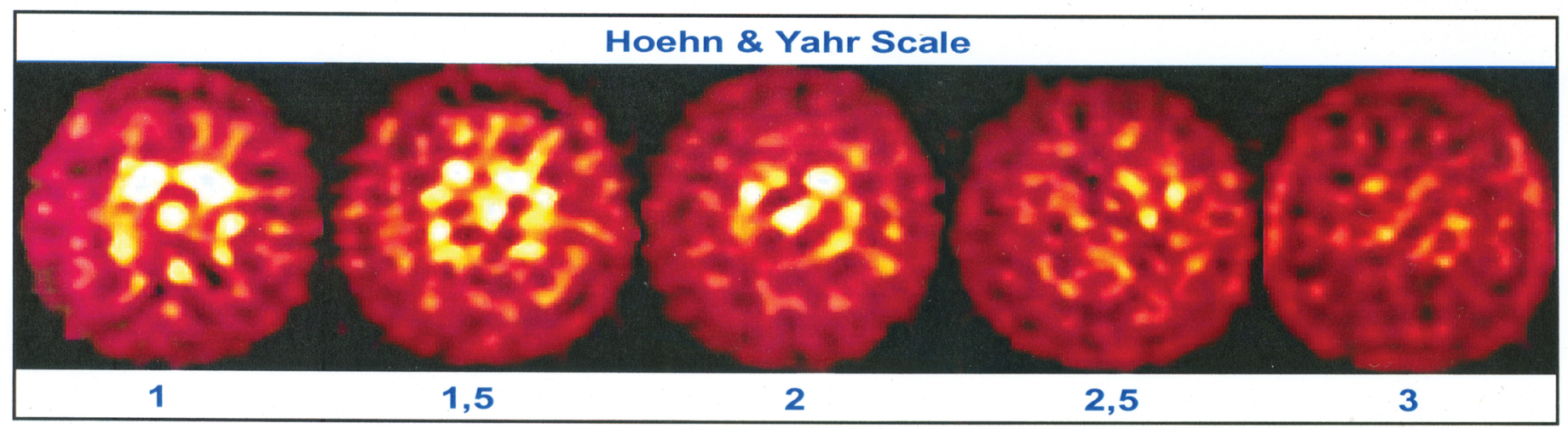

Figure $1-\left[{ }^{99 \mathrm{~m}} \mathrm{Tc}\right]-$ TRODAT-1 SPECT images at the level of the striatum from patients with increasing PD severity measured with Hoehn \& Yahr scale (higher color intensity means higher DAT density)

makes it difficult to differentiate PD from other movement disorders, such as essential tremor, especially at early stages of PD. Studies using TRODAT and SPECT have shown impressive diagnostic accuracy, with specificity higher than $97 \%$ and sensitivity greater than $91 \%$.

The higher DAT decrease in PD is in fact related to an advanced stage of neuronal loss. DAT loss observed in SPECT and PET can be seen even before the beginning of symptoms, since clinical manifestations take place after more severe dopamine neuron deterioration.

Another important example of the potential clinical utility of molecular imaging is the development of new radiotracers for Alzheimer's disease. Mounting efforts have been directed to the development of new treatment strategies to prevent or at least delay Alzheimer's disease progression through early interventions. Currently, combination of clinical and neuroanatomical imaging criteria provides reasonable sensitivity and specificity for the diagnosis of Alzheimer's disease only at mid or late stages but not at early stages. Molecular imaging radiotracers that bind to plaque and tangles can potentially identify Alzheimer's disease patients before clinical manifestation of the disease. Recent studies using a PET radiotracer ([$\left.\left.{ }^{18} \mathrm{~F}\right] \mathrm{FDDNP}\right)$ for plaque and tangles were able to differentiate among patients with Alzheimer's disease, mild cognitive impairment and healthy controls. ${ }^{4}$ This is one of the first studies to identify patients with mild cognitive impairment, who are at a transitional stage between normal aging and Alzheimer's disease. However, this diagnosis refers to a presumed underlying pathophysiological state, but the course of the condition is heterogeneous. Therefore, more studies validating the tracer are necessary to fully implement the method in clinical settings.
In psychiatric disorders such as schizophrenia, molecular imaging techniques have provided some of the most relevant pathophysiological evidence of the dopaminergic dysfunction in vivo in drug free patients (for review see Bressan et al., 2001). ${ }^{5}$ Initial efforts focused on $D_{1}$ and $D_{2}$ receptor density changes, but failed to show differences between patients and controls. More recently, the research groups endeavored dopamine function a more relevant challenge. They have produced several studies showing increased DOPA decarboxylase activity using $\left[{ }^{18} \mathrm{~F}\right]-\mathrm{FD} o p a$ or $\left[{ }^{11} \mathrm{C}\right]$-dopa, higher dopamine release after using amphetamine challenges and $\left[{ }^{11} \mathrm{C}\right]$-raclopride or $\left[{ }^{123} \mathrm{l}\right]$ IBZM, and higher concentration of dopamine in the synaptic cleft using alpha-methyl-paratyrosine challenges and [123|]IBZM. ${ }^{5}$ Although the findings are extremely relevant, demonstrating an increased dopamine activity in schizophrenia, these methods have not developed into biomarkers due to the high complexity of the disorder and low sensitivity and specificity of the findings.

Despite their considerable promise for application in the investigation of NPD, biomarkers still have limited utility in clinical practice. Recent studies are using a more powerful strategy combining several biological markers at the same time, including genetics, structural neuroimaging, neuropsychology, neuroendocrinology, and molecular imaging. This strategy can provide hundreds of thousands of variables, and some combinations of variables may provide more robust biomarkers to be used in clinical practice. These biomarkers may identify the underlying biological processes of NPD before the onset of symptoms and offer the opportunity to prevent the occurrence of devastating disorders. 
Rodrigo A Bressan, Ming C Shih Laboratório Interdisciplinar de Neurociências Clínicas (LiNC), Universidade Federal de São Paulo (UNIFESP), São Paulo (SP), Brazil Instituto Israelita de Ensino e Pesquisa Albert Einstein (IIEP-HIAE), São Paulo (SP), Brazil

Marcelo Q Hoexter Laboratório Interdisciplinar de Neurociências Clínicas (LiNC), Universidade Federal de São Paulo (UNIFESP), São Paulo (SP), Brazil

Acioly L T Lacerda Laboratório Interdisciplinar de Neurociências Clínicas (LiNC), Universidade Federal de São Paulo (UNIFESP),

São Paulo (SP), Brazil SINAPSE Institute, Campinas (SP), Brazil

Financing: This study was supported by a research grant from Instituto Israelita de Ensino e Pesquisa do Hospital Israelita Albert Einstein (IIEP-HIAE) and through collaboration between the Institute of Nuclear Energy (Taiwan, ROC) and the Universidade Federal de São Paulo (UNIFESP). R.A. Bressan is supported by a research grant from CAPES Foundation (Brazilian Government). M.C. Shih is supported by a PRO-DOC scholarship from CAPES Foundation.

Conflict of interests: None

\section{References}

1. Ravina B, Eidelberg D, Ahlskog JE, Albin RL, Brooks DJ, Carbon M, Dhawan V, Feigin A, Fahn S, Guttman M, Gwinn-Hardy K, McFarland H, Innis R, Katz RG, Kieburtz K, Kish SJ, Lange N, Langston JW, Marek K, Morin L, Moy C, Murphy D, Oertel WH, Oliver G, Palesch Y, Powers W, Seibyl J, Sethi KD, Shults CW, Sheehy P, Stoessl AJ, Holloway R. The role of radiotracer imaging in Parkinson disease. Neurology. 2005;64(2):208-15.

2. Shih MC, Amaro E Jr, Ferraz HB, Hoexter MQ, Goulart FO, Wagner J, Lin LF, Fu YK, Mari JJ, Lacerda AL, Tufik S, Bressan RA. [Neuroimaging of the dopamine transporter in Parkinson's disease: first study using [99mTc]-TRODAT-1 and SPECT in Brazil]. Arq Neuropsiquiatr. 2006;64(3A):628-34

3. Shih MC, Franco de Andrade LA, Amaro E Jr, Felicio AC, Ferraz HB, Wagner J, Hoexter MQ, Lin LF, Fu YK, Mari JJ, Tufik S, Bressan RA. Higher nigrostriatal dopamine neuron loss in early than late onset Parkinson's disease? A [(99m)Tc]-TRODAT-1 SPECT study. Mov Disord. 2007; [Epub ahead of print].

4. Small GW, Kepe V, Ercoli LM, Siddarth P, Bookheimer SY, Miller KJ, Lavretsky H, Burggren AC, Cole GM, Vinters HV, Thompson PM, Huang SC, Satyamurthy N, Phelps ME, Barrio JR. PET of brain amyloid and tau in mild cognitive impairment. $N$ Eng/ J Med. 2006;355(25):2652-63.

5. Bressan RA, Bigliani V, Pilowsky LS. Neuroimaging of D2 dopamine receptors in schizophrenia. Rev Bras Psiquiatr. $2001 ; 23$ (Suppl 1):46-9. 